\title{
ATUAÇÃO DO ENFERMEIRO COM PACIENTES COM DIABETES MELLITUS NA MELHORIA DA QUALIDADE DE VIDA
}

\author{
NURSES' ROLE IN THE IMPROVEMENT OF DIABETES MELLITUS PATIENTS' QUALITY OF LIFE
}

\author{
Josilene Araujo Rodrigues ${ }^{\mathrm{a}^{*}}$, Francimara de Jesus Sousa Lima ${ }^{\mathrm{b}^{*}}$, Ariane Gomes dos Santos ${ }^{\mathrm{c}^{*}}$

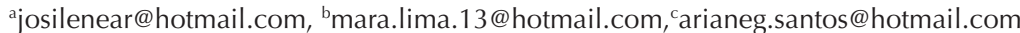 \\ *Instituto de Ensino Superior Múltiplo - Timon (MA), Brasil.
}

Data de recebimento do artigo: 08/12/2014 Data de aceite do artigo: 21/05/2015

\section{RESUMO}

Objetivo: Analisar as condiçóes que afetam a qualidade de vida de pacientes com diabetes mellitus conforme suas limitaçôes. Materiais e métodos: Trata-se de uma revisão integrativa da literatura, a coleta de dados foi realizada no período de fevereiro a agosto de 2014. Utilizou-se os descritores qualidade de vida, diabetes mellitus e enfermagem. A amostra final constituiu-se por 7 (sete) artigos pertencentes às bases de dados LILACS, BENDENF e MEDLINE, com recorte temporal entre os anos 2009 a 2014. A análise e discussão dos dados procederam-se de forma descritiva. Resultados: Os conteúdos dos artigos selecionados foram agrupados em três categorias analíticas: "fatores e aspectos associados ao diabetes que interferem na qualidade de vida", "dificuldades de adesão ao tratamento" e "conhecimentos da enfermagem sobre açóes desenvolvidas no atendimento dos pacientes diabéticos". O estudo evidenciou que muitas pessoas diabéticas não possuem conhecimento sobre a cronicidade e as inúmeras situaçóes impostas pela doença, há dificuldades de adesão aos elementos que fazem parte da terapêutica: o tratamento medicamentoso, exercício físico e plano alimentar. Estudos mostraram, ainda, que os enfermeiros possuem competência interpessoal, conhecimento, habilidade e capacidade para interagir com o paciente, aceitando seus problemas, crenças e desejos. Consideraçóes finais: Para que sejam atendidas as expectativas em relação ao tratamento, controle da doença e uma qualidade de vida digna aos portadores de diabetes, os enfermeiros têm a missão de desenvolver estratégias que convençam o paciente a aderir ao tratamento, a mudar seu estilo de vida, conscientizá-los sobre a patologia, e desenvolver o autocuidado.

Palavras-chave: Qualidade de vida; diabetes mellitus; enfermagem.

\section{ABSTRACT}

Objective: To analyze the conditions that affect the quality of life of patients with diabetes mellitus according to their limitations. Materials and Methods: This is an integrative literature review, data collection carried out from February to August 2014. We used the descriptors "quality of life", "diabetes mellitus" and "nursing". The final sample consisted of 7 (seven) items belonging to the databases LILACS, MEDLINE and BENDENF with time frame between 2009 to 2014. The analysis and discussion of the data proceeded descriptively. Results: The contents of the selected articles were grouped into three analytical categories: "factors and aspects associated with diabetes that affect quality of life","compliance to the treatment" and "nursing knowledge about actions developed in care of diabetic patients". The study showed that many people with diabetes are unaware about the chronicity and the numerous situations imposed by the disease, there are adhesion problems for elements that are part of therapy: drug treatment, exercise and eating plan. Studies also showed that nurses have interpersonal skills, knowledge, skill and ability to interact with the patient, accepting their problems, beliefs and desires. Final thoughts: To meet expectations with respect to treatment, disease control and a decent quality of life for people with diabetes, nurses have the mission to develop strategies to convince the patients to adhere to treatment, change their life style, make them aware of the disease, and develop self-care.

Keywords: Quality of life; diabetes mellitus; nursing. 


\section{Introdução}

O diabetes mellitus (DM) é um grupo de doenças metabólicas caracterizadas por aumento dos níveis glicêmicos, resultante de defeito na secreção e/ou ação da insulina.Essacondição crônica faz com que os indivíduos tenhamlimitaçôes em suas atividades diárias, devido às comorbidades que algumas pessoas podem apresen$\operatorname{tar}$ (dislipidemia, hipertensão arterial e disfunçáo endotelial), contribuindo dessa forma para diminuição da autoestima ${ }^{1}$.

A taxa de mortalidade causada pelo diabetes chega a cerca de 50 mil pessoas por ano, sendo que em 2010, 54 mil brasileiros morreram em consequência do diabetes.Essa patologia afeta mais de 200 milhôes de pessoas no mundo atual, e estima-se que em 2025 o número de diabéticos seja de 380 milhões. A doença apresenta altos índices de novos casos e mortalidade, além de ter significante custo social e financeiro para a sociedade e os sistemas de saúde 2 .

Além disso, o diabetes pode afetar a qualidade de vida $(\mathrm{QV})$ de seus portadores, sendo esta um elemento específico de avaliação de aspectos relacionados às repercussôes da saúde, doença e tratamento. É importante evidenciar a necessidade de um tratamento que tenha repercussóes não só na esfera biológica, mas também na psicossocial, visando à adesão ao tratamento e o bem-estar do paciente ${ }^{3}$.

A QV é formada por muitos fatores, dentre eles: o bem-estar individual, a satisfação nas relaçôes sociais, ambientais e culturais etc. Esses fatores dependem do conhecimento do indivíduo, do lugar onde ele vive, do grupo de convívio social e das esperanças próprias em relação a conforto e bem-estar.É importante buscar qualidade de vida frente às possibilidades de cada indivíduo por meio da autonomia da sua própria vida, enfatizando-se que bem-estar e qualidade de vida são sinônimos ${ }^{4}$.

É importante considerar os aspectos físicos e a autonomia como fatores de impacto na vida dos doentes, interferindo principalmente na manutenção de suas atividades com a comunidade, bem como buscar alternativas para melhoria da qualidade de vida ${ }^{5}$.

A enfermagem pode promover ações que visemà manutenção da autonomia de pacientes diabéticos, além de atividades direcionadas para o cuidado com a saúde. Visa-se potencializar os aspectos físicos, por vezes minimizados pelos efeitos decorrentes da doença. A atenção à saúde dos diabéticos deve ser estruturada de modo a incentivar o apoio familiar, contribuindo para a participação social e melhoria dos aspectos psicológicos 5 . O acolhimento é um desafio na construção da integralidade do cuidado, sendo uma rede de confiança e solidariedade entre usuários, profissionais e equipes de saúde que beneficie a edificação de uma relação de confiança e respeito com aquele que busca o atendimento para satisfazer suas necessidades ${ }^{6}$.

A realização do estudo faz-se relevante pelo fato do diabetes mellitus ser uma doença de elevada morbidade e que, se náo tratada, pode causar incapacidades, as quais podem afetar a qualidade de vida dos indivíduos acometidos. Além disso, poderá despertar o interesse dos profissionais de saúde, principalmente enfermeiros, sobre a importância do acolhimento humanizado, a fim de proporcionar aos pacientes e familiares uma melhor qualidade de vida diante das adversidades que a doença crônica pode desencadear.Os objetivos deste estudo foram analisar as condiçóes que afetam a qualidade de vida de pacientes com diabetes mellitus conforme suas limitaçôes, descrever o papel do enfermeiro no acolhimento aos portadores de diabetes mellitus e verificar a adesão do diabético ao tratamento terapêutico.

\section{Materiais e métodos}

Trata-se de uma revisão integrativa da literatura, com abordagem qualitativa. A revisão integrativa é o mais amplo tipo de revisão da literatura, permitindo a inclusão de estudos experimentais e não experimentais para uma compreensão completa do fenômeno analisado. Combina também dados da literatura teórica e empírica, além de incorporar um vasto leque de propósitos: definiçãao de conceitos, revisão de teorias, evidências e análise de problemas metodológicos de um tópico particular $^{7}$.

Para realização dessa revisão foram seguidos os passos de: identificação do tema e seleção da hipótese; amostragem; categorização dos estudos; avaliação dos estudos; interpretação dos resultados e síntese do conhecimento. $\mathrm{O}$ estudo foi guiado pela questão norteadora: qual a importância da atuação do enfermeiro na melhoria da qualidade de vida de pacientes com diabetes mellitus?

A coleta de dados foi realizada no período de fevereiro a agosto de 2014, nas bases de dados da Literatura Latino-Americana e do Caribe em Ciências da Saúde (LILACS), Base de Dados de Enfermagem (BDENF) e Literatura Internacional em Ciências da Saúde (MEDLINE). Utilizaram-se os seguintes descritores: qualidade de vida, diabetes mellitus e enfermagem, todas de acordo com BIREME. Para sistematizar a pesquisa foi utilizado o operador booleanoAND, tendo como recorte temporal os anos de 2009 a 2014.

Após o levantamento das publicaçóes, os resumos foram lidos e analisados segundo os critérios de inclusão e exclusão preestabelecidos. Como critérios de inclusão foram utilizadosartigos completos (em português e inglês) disponíveis eletronicamente e publicados no período de 
janeiro de 2009 a junho de 2014 que retratam a atuação do enfermeiro na melhoria da qualidade de vida de pacientes com diabetes mellitus (e que responderam à questáo norteadora deste estudo).Optou-se por excluir artigos que náo estivessem disponíveis na íntegra, teses, dissertaçóes e estudos de revisão de literatura.

Após a pesquisa, por meio dos descritores estabelecidos, foram encontrados 52 artigos, no entanto, após aplicação dos critérios de inclusão e exclusão, apenas sete artigos foram selecionados e compuseram a amostra final do estudo.

Para a coleta de dados, foi utilizado um instrumento desenvolvido pelos autores, o qual contemplou as seguintes variáveis: autor, título, abordagem, tipo de estudo, periódico, ano de publicação e o objetivo de cada estudo.

A análise dos estudosprocedeu-se de forma descritiva, possibilitando observar, analisar e correlacionar os dados com a maior precisão possível. Os resultados foram agrupadosem categorias analíticas de acordo com as temáticas abordadas.

\section{Resultados e discussão}

Os sete artigos selecionados foram analisados na íntegra e, ao observar o predomínio de pesquisa em anos mais recentes, três artigos foram encontrados (o correspondente a $42,8 \%$ nos anos de 2012 e 2013 ), todos pertencentes à área de enfermagem. Quanto à abordagem metodológica das pesquisas, foram observados cinco estudos $(71 \%)$ com abordagem quantitativa e dois estudos (28\%) com abordagem qualitativa. Quanto ao tipo de estudo, a maior parte dos selecionados eram transversais, representados por $57,1 \%$ dos artigos.

Quadro 1: Perfil dos estudos encontrados de acordo com autores, ano de publicação, periódico, abordagem e objetivos.

\begin{tabular}{|c|c|c|c|c|}
\hline Autores & Ano & Periódico & $\begin{array}{l}\text { Tipo de estudo/ } \\
\text { Abordagem }\end{array}$ & Objetivos \\
\hline $\begin{array}{l}\text { Ferreira LE, ZanattaEA, } \\
\text { Brum MLB, Nothaft SC, } \\
\text { Motta MGC } \\
\end{array}$ & 2013 & CogitareEnferm & $\begin{array}{l}\text { Estudo etnográfico/ } \\
\text { Qualitativa }\end{array}$ & $\begin{array}{l}\text { Conhecer percepçóes e identificar limitaçóes que esses } \\
\text { vivenciam devido à doença no âmbito familiar, social e } \\
\text { alimentar. }\end{array}$ \\
\hline $\begin{array}{l}\text { Faria HTG, Rodrigues } \\
\text { FFL, Zanetti ML, Araújo } \\
\text { MFM, Damasceno MMC }\end{array}$ & 2013 & $\begin{array}{l}\text { Acta Paul } \\
\text { Enferm }\end{array}$ & $\begin{array}{l}\text { Estudo transversal/ } \\
\text { Quantitativa }\end{array}$ & $\begin{array}{l}\text { Investigar associação entre adesão ao tratamento do diabe- } \\
\text { tes mellitus tipo } 2 \text { e variáveis sociodemográficas, clínicas e } \\
\text { controle metabólico. }\end{array}$ \\
\hline $\begin{array}{l}\text { Alves TOS, Souza SA, } \\
\text { Souza ECS, Gois CFL, } \\
\text { Guimarães AMDN, Mattos } \\
\text { MCT }\end{array}$ & 2013 & $\begin{array}{l}\text { Rev Min } \\
\text { Enferm }\end{array}$ & $\begin{array}{l}\text { Estudo transversal/ } \\
\text { Quantitativa }\end{array}$ & $\begin{array}{l}\text { Evaluate the } \\
\text { Health-related quality of life (HRQOL) of people with } \\
\text { diabetes mellitus and its relation to gender, age, time spent } \\
\text { since the diagnosis of diabetes, and the practice of physical } \\
\text { activity. }\end{array}$ \\
\hline $\begin{array}{l}\text { Cardoso GM, ValoesLMA, } \\
\text { Almeida OAE, Ferrari CKB }\end{array}$ & 2012 & $\begin{array}{l}\text { Enfermagem em } \\
\quad \text { Foco }\end{array}$ & $\begin{array}{l}\text { Estudo transversal/ } \\
\text { Quantitativa }\end{array}$ & $\begin{array}{l}\text { Avaliar a qualidade de vida (QV) e a percepção da doença } \\
\text { em pacientes com diabetes mellitus (DM) atendidos na } \\
\text { Estratégia de Saúde da Família (ESF) em Aragarças-GO. }\end{array}$ \\
\hline $\begin{array}{l}\text { Braz JM, Silva MR, Gois } \\
\text { CFL, Braz TM, Santos V, } \\
\text { Silva LASM }\end{array}$ & 2012 & Rev Rene & $\begin{array}{l}\text { Estudo descritivo/ } \\
\text { Quantitativa }\end{array}$ & $\begin{array}{l}\text { Averiguar a associação entre sintomas depressivos e variá- } \\
\text { veis sociodemográficas e clínicas, e adesão ao tratamento } \\
\text { medicamentoso em pessoas com diabetes mellitus tipo } 2 \\
\text { (DM2). }\end{array}$ \\
\hline $\begin{array}{l}\text { Lessmann JC, Silva } \\
\text { DMGV, Nassar SM }\end{array}$ & 2012 & $\begin{array}{l}\text { Acta Paul } \\
\text { Enferm }\end{array}$ & $\begin{array}{l}\text { Estudo transversal/ } \\
\text { Quantitativa }\end{array}$ & $\begin{array}{l}\text { Conhecer o perfil de mulheres com Diabetes mellitus tipo } \\
2 \text {, enfocando fatores sociodemográficos, biométricos e de } \\
\text { saúde }\end{array}$ \\
\hline $\begin{array}{l}\text { Soares D A, Sadigursky D, } \\
\text { Soares, I }\end{array}$ & 2011 & RevBrasEnferm & $\begin{array}{l}\text { Estudo descriti- } \\
\text { vo exploratório/ } \\
\text { Qualitativa. }\end{array}$ & $\begin{array}{l}\text { Apreender a percepção de enfermeiros que cuidam de } \\
\text { pessoas com Diabetes sobre a competência interpessoal. }\end{array}$ \\
\hline
\end{tabular}

Em decorrência das análises, três categorias analíticas emergiram: "fatores e aspectos associados ao diabetes que interferem na qualidade de vida", "dificuldades de adesão ao tratamento" e "conhecimentos da enfermagem sobre açôes desenvolvidas no atendimento dos pacientes diabéticos". 


\section{Fatores e aspectos associados ao diabetes que interferem na qualidade de vida}

Três artigos $(42,8 \%)$ fizeram parte desta categoriae relataram sobre conscientização dos diabéticos em relação à doença, algumas patologias associadas e comprometimento da qualidade de vida ${ }^{8,9,10}$.

Estudo realizado na região Sudeste do Brasil evidenciou que outras patologias estão associadas ao DM, como a hipertensão arterial e as complicaçóes crônicas (retinopatias e cardiopatias), agravos estes que prejudicam aQV de seus portadores ${ }^{8}$.

O DM é uma patologia com tendências a complicações agudas e crônicas. Deve-se, dessa forma, atentar para essas adversidades com açóes preventivas, pois com isso, haverá uma diminuição da incapacidade funcional do paciente e incentivo à autonomia, à independência $\mathrm{e}$ à melhoria na $\mathrm{QV}^{11}$.

Ao avaliar a acuidade visual dos pacientes, deve-se atentar à retinopatia (uma complicação crônica decorrente do DM,que deve ser tratada o mais precocemente possível), como também, aos problemas circulatórios e cardíacos que são agravantes e resultam em outras complicaçóes, como o aparecimento de úlceras em membros inferiores e hipertensão $\operatorname{arterial}^{12}$.

Assim, faz-se necessário uma avaliação precisa da condição circulatória periférica, a fim de prevenir possíveis lesóes e a condição cardíaca que, por sua vez, desencadeia a hipertensão arterial e outros acometimentos, exigindo uma maior atençáo aos portadores de DM no que diz respeito aos hábitos de vida, ao uso correto das medicaçóes e à assiduidade nas consultas avaliativas ${ }^{12}$.

Estudo realizado em Aragarças (GO) mostrou que as pessoas diabéticas que possuem consciência da cronicidade e das inúmeras situações impostas pela doençatêm melhor estado físico, social, emocional e consequentemente melhor qualidade de vida?

Em estudo realizado no Ambulatório de Endocrinologia da Universidade Federal de Sergipe evidenciou-se que a comorbidade que mais prevaleceu nas pessoas com diabetes foia hipertensão arterial, e a associação entre ambas aumentou o risco para doenças cardiovasculares ${ }^{10}$.

A prevalência de hipertensão arterial é maior nas pessoas com DM. Outros fatores também podem estar presentes, como obesidade, sedentarismo, idade avançada, dislipidemia, tabagismo e histórico familiar ${ }^{13}$.Para prolongar ou impedir o aparecimento de complicaçóes crônicas faz-se necessário manter os níveis glicêmicos dentro dos padróes de normalidade, mas, para que isso aconteça, é preciso associar semestralmente as mediaçôes prescritas, o controle da dieta, exercícios físicos regulares e exames laboratoriais, pois o tratamento isolado não proporciona um bom controle glicêmico ${ }^{14}$.

\section{Dificuldades de adesão ao tratamento}

Nessa categoria enquadraram-se quatro artigos (57\%). Estes demonstraram as dificuldades dos pacientes diabéticos de praticarem atividade física, seguirem o plano alimentar e aderirem ao tratamento medicamentosopara controlar a doença ${ }^{8,15,16,17}$.

Um estudo realizado no oeste de Santa Catarina mostrou as dificuldades e limitaçóes impostas pela doença como as restriçốes alimentares ${ }^{15}$.O descontrole metabólico é fator de risco para as complicações relacionadas à doença. É necessáriaa implementação de medidas educativas para prevenção e controle da doença. A educação em saúde deve transmitir informaçóes-chave ao indivíduo para que este desenvolva habilidades para o controle da doença, promovendo um estilo de vida saudável ${ }^{18}$.

Muitos diabéticos não possuem o hábito de praticarem regularmente atividade física, hábito que prejudica o tratamento ${ }^{19}$. Sintomas de mal estar seguido dos valores glicêmicos alterados prejudicam a realização de atividades da vida diária. Outra dificuldade é aceitar as cobranças do tratamento, a exatidão nos horários das medicaçóes, o cumprimento de dosagens glicêmicas diariamente e convívio com as cobranças dos profissionais ${ }^{15}$.

Em estudo realizado na região Sudeste do Brasil, foram elucidadasas dificuldades dos indivíduos portadores de diabetes mellitus para aderirem ao tratamento medicamentoso, ao exercício físico e ao plano alimentar. O estudo evidenciou que aproximadamente 50\% aderiram a dois elementos, sendo que a maior parte dos diabéticos aderiu a um único e a minoria fez associação entre os três elementos ${ }^{8}$.

$\mathrm{O}$ método de avaliação da aderência à terapêutica requer atenção, aceitação da doença, adesão às modificações no estilo de vida, como: dieta apropriada, realização de exercícios físicos, adotar medidas de autocuidado e abandonar hábitos como o alcoolismo e tabagismo. Além disso, a falta de conhecimento do paciente sobre a doença e suas possíveis complicaçóes colabora expressivamente para a não aderência ao tratamento ${ }^{14}$.

A educação em saúde é um método capaz de adequar maior adesão à terapêutica. Assim, o paciente informado sobre terapia nutricional e sua importância é influenciado a ter novas práticas e mudanças no seu estilo de vida, facilitando o tratamento e, consequentemente, evitando implicaçóes futuras causadas pelo descontrole glicêmico ${ }^{20}$.

Em pesquisa realizada no Hospital Universitário da Universidade Federal de Sergipe (UFS), revelou-se que poucos dos indivíduos com diabetes realizavam atividade física semanalmente, como hidroginástica, dança ou caminhada; e a terapêutica medicamentosa mais citada 
eram os hipoglicemiantes orais associados à insulina ${ }^{16}$. No entanto, pesquisa realizada em Florianópolis constatou que pessoas com diabetes conseguem seguir adequadamente as recomendaçóes alimentares como: a restrição do consumo de carboidratos e lipídios, realizaçáo de atividades físicas e, com isso, manutençãoda glicemia e do peso corporal dentro dos padróes de normalidade ${ }^{17}$. Nota-se, dessa forma, que o sucesso do tratamento do DM depende da conscientizaçáo do paciente quanto à importância daquele.

Após o diagnóstico, a pessoa com diabetes reage com ansiedade, medo, nervosismo, revolta e aflição, gerando uma variedade de respostas emocionais entre aceitação e resistência. Além disso, muitas vezes os pacientes têm resistência quanto ao uso da insulina, por ser uma droga injetável e um método invasivo, doloroso e realizado constantemente. Nesse momento, devem receber informaçóes da equipe de saúde sobre a patologia, as terapêuticas utilizadas e as consequências da doença ${ }^{21,22}$.

\section{Conhecimento da enfermagem sobre ações desenvolvidas no atendimento dos pacientes diabéticos}

Essa categoria foi composta por dois artigos (28\%), os quais discorreram sobrea assistência de enfermagem a pacientes com diabetes ${ }^{23,15}$.

Um estudo realizado em ummunicípio do Sudoeste Baiano mostrou que os enfermeiros possuem competência interpessoal, conhecimento, habilidade e capacidade para interagir com o paciente, aceitando seus problemas, crenças e desejos. Esses profissionais têm como elementos imprescindíveis a confiança e o respeito, e assim, buscam harmonização dos interesses e expectativas de ambos os envolvidos no processo do cuidar nas consultas, visitas domiciliares ou grupos de educação em saúde ${ }^{23}$.

Algumas estratégias devem ser utilizadas para uma a adesão mais efetiva dos pacientes ao tratamento, os profissionais de enfermagem precisam ultrapassar o modelo de atençáo medicamentosa, tendo um olhar mais amplo sobre a terapêutica estabelecida. Com isso, devem contemplar aspectos envolvidos no cuidado dos pacientes como os fatores culturais e colaborar para as mudanças dos hábitos de vida não saudáveis e dos costumes e crenças errôneas sobre a doença e tratamento ${ }^{24}$.

$\mathrm{O}$ enfermeiro tem seu papel voltado para a educação em saúde em caráter mais detalhado e aprofundado para a averiguação das necessidades de bem-estar e efetivação das atividades assistenciais em benefício das pessoas diabéticas ${ }^{25}$. Considerando o DM uma doença crônica, é evidente a necessidade de atuação da enfermagem para proporcionar a adoção de estratégias de transformaçóes no comportamento dos portadores da patologia ${ }^{26}$.

Os profissionais que lidam com diabéticos precisam refletir sobre as práticas educativas para edificar o autocuidado, devendo procurar desenvolver habilidades na educação em saúde, admitir as dificuldades e as limitaçóes, permitindo melhorar a prática educativa para o autocuidado do paciente ${ }^{18}$. O papel do enfermeiro passou do simples fato de orientar para o de favorecer e conscientizar os sujeitos sobre o modo que vivem e as implicações de suas escolhas para a saúde. Dessa forma, o enfermeiro deve desenvolver sua profissão com uma visão holística, com compromisso e responsabilidade para com o ser humano ${ }^{27}$.

Um estudo desenvolvido no Oeste de Santa Catarina mostrou que as intervenções de enfermagem necessitam ser dialogadas, no sentido de acolher e entender o que se passa com todos os pacientes e familiares, assim, incentivam a aceitação do indivíduo quanto ao convívio com a doença. $\mathrm{O}$ planejamento e realização das intervençóes devem ser realizados levando-se em consideração aspectos individuais, sociais, culturais e coletivos ${ }^{15}$.

Cuidar de uma pessoa com diabetes requer dos enfermeiros capacidades e conhecimentos específicos sobre a doença, como identificação de sinais, sintomas eoutraspeculiaridades, para orientar o paciente e a sua família sobre o estado de sua saúdee esclarecerdúvidas. Assim, a assistência será realizada com segurança e autonomia, pois informaçóes superficiais ou baseadas somente em termos técnicosimpossibilitam o paciente e a família de manter o autocuidado e controle da doença ${ }^{28}$. Um trabalho do enfermeiro com a família e sociedade torna as pessoas com diabetes mais seguras diante dos problemas enfrentados, com melhor aceitação da doença e maior adesão ao tratamento proposto ${ }^{22}$.

\section{Considerações finais}

Este estudo demonstrou os fatores e aspectos associados ao diabetes que interferem na qualidade de vida dos seus portadores, as dificuldades de adesão ao tratamento e conhecimentos da enfermagem sobre açoos desenvolvidas no atendimento aos pacientes diabéticos.

Sendo o diabetes uma doença que pode apresentar complicaçóes agudas e crônicas, é importante que o paciente tenha consciência de suas limitaçóes e que faça mudanças no seu estilo de vida, além do controle glicêmico da doença. Sabe-se também que a falta de conhecimento do paciente atrelada à falta de qualificação profissional adequada do enfermeiro são obstáculos para o alcance da melhoria da qualidade de vida de diabéticos.

A adesão ao tratamento é um fator importantíssimo para a melhoria qualidade de vida. Como complemento 
ao tratamento, a família tem um papel significativo na vida de uma pessoa com diabetes, pois deve apoiar e incentivar o paciente para melhor adesão ao tratamento e mudanças de hábitos na sua rotina.

Para que sejam atendidas as expectativas em relaçáo ao tratamento, controle da doença e uma qualidade de vida digna aos portadores de diabetes, a equipe de saúde deve conhecer a doença e estar habilitada a lidar com ela, e essa exigência recai, principalmente, sobre a equipe de enfermagem, pois tais profissionais somam atribuiçóes, não sendo apenas agentes de saúde, mais orientadores e educadores no processo de cuidar. Os enfermeiros têm a missão de desenvolver estratégias como atividades educativas que convençam o paciente a aderir ao tratamento, a mudar seu estilo de vida, sensibilizá-los sobre a patologia, a desenvolver melhor o autocuidado com sua saúde.

Como limitação observou-se que poucos dados encontravam-se disponíveis nas bases de dados pesquisadas, sobre a qualidade de vida dos portadores de diabetes, principalmente no que se refere à atuação da enfermagem. Assim, faz-se necessária a realização de novos estudos que abordem sobre a temática. Vale ressaltar, no entanto, que a limitação apresentada não diminui a relevância do estudo realizado.

\section{Referências}

1. Grossi AS, Pascali PM. Cuidados de enfermagem em diabetes melittus. Sociedade Brasileira de Diabetes. Departamento de Enfermagem da Sociedade Brasileira de Diabetes. SãoPaulo, 2009. [acesso em 14 mar. 2014]. Disponível em: <http://www.diabetes.org.br/attachments/1118_1324_manual_enfermagem.pdf >.

2. Brasil. Ministério da Saúde. Brasil produzirá insulina humana a partir de 2016. Brasília: Ministério da Saúde; 2013.

3. Novato TS, Grossi SAA. Fatores associados à qualidade de vida de jovens com diabetes mellitus do tipo 1. Rev Esc Enferm. 2011;45(3):770-6.

4. Almeida MAB, Gutierrez GL, Marques R. Qualidade de vida: definição, conceitos e interfaces com outras áreas, de pesquisa. In: Almeida MAB, Gutierrez GL, Marques R. Qualidade de vida: uma área de conhecimento em processo de definição. São Paulo: Escola de Artes, Ciências e Humanidades; 2012. p.15-17.

5. Tavares DMS, Côrtes RM, Dias FA. Qualidade de vida e comorbidades entre os idosos diabéticos. Rev Enferm. 2010;18(1):97-103.

6. Coelho MO, Jorge MSB, Araújo MEO. Acesso por meio do acolhimento na atenção básica à saúde. Rev Saúde Pública. 2009;33(3):440-52.

7. Souza MT, Silva MD, Carvalho R. Revisão integrativa: o que é e como fazer. Einstein. 2010;8(1):102-6.
8. Faria HTG, Rodrigues FFL, Zanetti ML, Araújo MFM, Damasceno MMC. Fatores associados à adesão ao tratamento de pacientes com diabetes mellitus. Acta Paulist Enferm. 2013;26(3):231-7.

9. Cardoso GM, Valoes LMA, Almeida OAE, Ferrari CKB. Qualidade de vida na percepção da gravidade da doença em portadores de diabetes mellitus. Enfermagem em Foco. 2012;3(3):143-6.

10. Alves TOS, Souza SA, Souza ECS, Gois CFL, Guimarães AMDN, Mattos MCT. Health-related quality of life of people with diabetes mellitus. Rev Mineira Enferm. 2013;17(1):135-40.

11. Tavares DMS, Côrtes RM, Dias FA. Qualidade de vida de idosos com diabetes mellitus. Ciência Cuidado e Saúde. 2011;10(2):290-7.

12. Santos E A, Tavares DMS, Rodrigues LR, Dias FA, Ferreira PCS. Morbidades e qualidade de vida de idosos com diabetes mellitus residentes nas zonas rural e urbana. Rev Esc Enferm. 2013;47(2):393-400.

13. Santos I, Guerra RG, Silva LA. Características individuais e clínicas de pessoas idosas com diabetes: investigação temática em oficina sociopoética. Rev Enferm. 2013;21(1):34-40.

14. Morais GFC, Soares MJGO, Costa MML, Santos IBC. O diabético diante do tratamento, fatores de risco e complicações crônicas. RevEnferm. 2009;17(2):240-5.

15. Ferreira LE, Zanatta EA, Brum MLB, Nothaft SC, Motta MGC. Diabetes mellitus sob a ótica do adolescente.Cogitare Enferm. 2013;18(1):71-7.

16. Braz JM, Silva MR, Gois CFL, Braz TM, Santos V, Silva LASM. Sintomas depressivos e adesão ao tratamento entre pessoas com diabetes mellitus tipo 2. Rev Rede Enferm Nordeste. 2012;13(5):1092-9.

17. Lessmann JC, Silva DMGV, Nassar SM. Mulheres com Diabetes mellitus tipo 2: perfil sociodemográfico, biométrico e de saúde. Acta Paulist Enferm. 2012;25(1):81-6.

18. Torres HC, Souza ER, Lima MHM, Bodstein RC. Intervençáo educativa para o autocuidado de indivíduos com diabetes mellitus. Acta PaulistEnferm. 2011;24(4):514-9.

19. Souza ECS, Souza AS, Alves TOS, Gois CFL, Guimarães AMDN, Mattos MCT et al. Avaliação da qualidade de vida de portadores de diabetes utilizando a medida específica B-PAID. Revista Mineira Enferm. 2012;16(4):509-14.

20. Meirelles ECD, Gomes PC. Compreendendo, sob a ótica do portador de diabetes, usuário do SUS o significado da alimentação no controle de sua doença. São Paulo: [trabalho de conclusão de curso]. Faculdade Santa Marcelina, 2011.

21. Borba AKOT, Marques APO, Leal MCC, Ramos RSPS. Práticas educativas em diabetes Mellitus: revisão integrativa da literatura. Rev Gaúcha de Enferm. 2012;33(1):169-76.

22. Ferreira DSP, Daher DV, Teixeira ER, Rocha IJ. Repercussão emocional diante do diagnóstico de diabetes mellitus tipo 2 . Rev Enferm. 2013;21(1):41-6.

23. Soares D A, Sadigursky D, Soares, I. Competência interpessoal no cuidado de pessoas com diabetes: 
percepção de enfermeiros. Rev Brasileira Enferm. 2011;64(4):677-83.

24. Rodrigues FFL, Santos MA, Teixeira CRS, Gonela JT, Zanetti ML. Relação entre conhecimento, atitude, escolaridade e tempo de doença em indivíduos com diabetes mellitus. Acta Paulist Enferm. 2012;25(2):284-90.

25. Kebian LVA, Acioli S. Visita domiciliar: espaço de práticas de cuidado do enfermeiro e do agente comunitário de saúde. Rev Enferm. 2011;19(3):403-9.
26. Marinho NB, Vasconcelos HC, Alves, Alencar AMPG, Almeida PC, Damasceno MMC. Risco para diabetes mellitus tipo 2 e fatores associados. Acta Paulist Enferm.2013;26(6):569-74.

27. Sousa LB. Práticas de educação em saúde no Brasil: a atuação da enfermagem. Rev Enferm. 2010;18(1):55-60.

28. Araújo YB, Collet N, Moura FM, Nóbrega RD. Conhecimento da família acerca da condição crônica na infância. Texto \& Contexto Enferm. 2009;18(3):498-505.

\section{Como citar este artigo:}

Rodrigues JA, Lima FJS, Santos AG. Atuação do enfermeiro com pacientes com diabetes mellitus na melhoria da qualidade de vida. Rev. Aten. Saúde. 2015;13(46):84-90 